\title{
Switched Stabilization of Higher-order Switched Linear Systems
}

\author{
Zhendong $\operatorname{Sun}^{\dagger}$ and S. S. Ge \\ ${ }^{\dagger}$ Hamilton Institute \\ National University of Ireland, Maynooth, Ireland \\ Email: zhendong.sun@nuim.ie \\ $\ddagger$ Department of Electrical and Computer Engineering \\ National University of Singapore, Singapore 117576 \\ Email: elegesz@nus.edu.sg
}

\begin{abstract}
In this paper, we investigate the underlying fundamental relation between switched controllability and switched stabilization for switched linear control systems. It is shown by a counterexample that switched controllability does not imply switched stabilization by means of piecewise linear state feedback controllers. In addition, piecewise linear state feedback stabilizable and controllable switched systems may not be quadratic stabilizable. To illustrate, we take third-order systems as a case study and identify conditions for quadratic stabilizability and non-quadratic stabilizability.
\end{abstract}

\section{Introduction}

Switched linear systems, which consist of sets of linear timeinvariant subsystems and switching signals that coordinate the switching among the subsystems, provide an attractive framework for modelling, analysis and design of complex dynamical systems. First, the switching structure represent a wide class of real-world systems with both continuous dynamics and discrete elements, such as power electronics, networks and digital control systems. Second switched linear systems can approximate nonlinear dynamics with arbitrary accuracy via linearizations at different operating points. Third, the intelligent structure of the two-level system representation is expected to enhance the robustness and adaptation to the changing and/or unknown parameters and uncertainties. Finally, the linearity of the subsystems enables us to analyze and design using tools from linear and multilinear theories. The reader is referred to the survey papers [1], [7] for recent development.

One of the central topics of switched linear control systems is the synthesis problem that derives stabilizing switching laws and feedback control laws for switched unstable systems. This topic attracts much attention as can be seen from the numerous publications in the literature. Among these, the existence of a stable convex combination of the subsystems can lead to an elegant construction of a stabilizing switching signal [11], [12]; conic switching laws were proposed to study second-order switched linear systems [3], [14]; and an observer-based switching strategy was developed to stabilize a class of controllable switched linear control systems [6].

In this paper, we investigate the underlying relationship between switched controllability and switched stabilizability for switched linear control systems. It is well-known that a completely controllable linear system is linear state feedback stabilizable. The relation still holds for lower (first or second) order switched linear systems [4], [8]. However, the relationship does not hold true for higher-order $(n \geq 3)$ switched linear systems, as shown in this paper. Moreover, even a switched controllable system is switched stabilizable, it may not be quadratically stabilizable. These make the problem of switched stabilization very challenging. In the sequel, the problem is handled as follows: the first step is to convert a controllable system into an equivalent system with triangular structure, the next step is classifying the systems into several cases each with a normal form, then verify case by case the stabilizability of the normal forms, and finally construct stabilizing switching/control laws. The first step has been addressed in [5], and the others will be done in this work by taking the third-order systems as case study. One merit of the approach is that it provides constructive solutions for the problem of switched stabilization including quadratic and non-quadratic stabilizabilities.

The paper is organized as follows. In Section II, we present preliminaries including system description, definitions and supporting lemmas. Main results are presented in Section III, while Section IV briefly concludes the work.

\section{Preliminaries}

\section{A. Systems Description}

Let $\bar{m}$ denote the set $\{1, \cdots, m\}$ for a natural number $m$.

Consider a switched linear control system given by

$$
\dot{x}(t)=A_{\sigma} x(t)+B_{\sigma} u(t) \quad x\left(t_{0}\right)=x_{0}
$$

where $x \in \mathbf{R}^{n}$ are the states, $u(t) \in \mathbf{R}^{p}$ is the controlled input, $\sigma \in \bar{m}$ is the controlled switching signal, and matrix pair $\left(A_{i}, B_{i}\right)$ for $i \in \bar{m}$ denotes a subsystem of (1).

In general, the switching signal $\sigma$ may depend on its past value, the time and the state

$$
\sigma(t)=\psi(t, x(t), \sigma(t-))
$$


or it may only depend on its past value and the state

$$
\sigma(t)=\psi(x(t), \sigma(t-)) \quad \forall t .
$$

In the latter case, the switching signal is said to be in statefeedback form. We assume that the switching signal is taken such that the switched system is well-posed. That is, for any initial state $x\left(t_{0}\right)=x_{0}$, the switched system always admits a solution for all forward time and there involve a finite number of switchings in any finite time interval. For a switching signal, any jump instant is said to be a switching time. That is, at a switching time $t$, we have $\lim _{s \uparrow t} \sigma(s) \neq \lim _{s \downarrow t} \sigma(s)$. The ordered sequence of the switching times is said to be the switching time sequence of the switching signal. Similarly, the ordered index sequence at the switching times is said to be the switching index sequence of the switching signal.

For clarity, let $\Sigma\left(A_{i}, B_{i}\right)_{\bar{m}}$ denote switched linear system (1), and $\phi\left(t ; t_{0}, x_{0}, \sigma\right)$ denote the state trajectory at time $t$ initialized at $x\left(t_{0}\right)=x_{0}$ via switching signal $\sigma$.

\section{B. Definitions}

Definition 1: State $x \in \mathbf{R}^{n}$ is (switched) controllable, if there exist a time instant $t_{f}>0$, a switching signal $\sigma:\left[0, t_{f}\right] \rightarrow \bar{m}$, and an input $u:\left[0, t_{f}\right] \mapsto \mathbf{R}^{p}$, such that $\phi\left(t_{f} ; 0, x, u, \sigma\right)=0$. The (switched) controllable set of system (1) is the set of states which are controllable. The system is said to be (switched) completely controllable, if its controllable set is $\mathbf{R}^{n}$.

Definition 2: System (1) is said to be (switched piecewise) linear (state) feedback stabilizable, if there exist a switching signal $\sigma$, and a piecewise linear state feedback control input

$$
u(t)=F_{\sigma} x(t)
$$

such that the closed-loop switched system

$$
\dot{x}(t)=\left(A_{\sigma}+B_{\sigma} F_{\sigma}\right) x(t)
$$

is uniformly asymptotically stable.

Note that, in the piecewise linear feedback control law (2), a linear feedback controller is associated to a subsystem. As a result, the closed-loop system is till a switched linear system.

Definition 3: System (1) is said to be (switched piecewise linear state) quadratically stabilizable, if there exist a switching signal $\sigma$ in state-feedback form, a piecewise linear state feedback control input in form (2), and a quadratic positive definite function $V(x)=x^{T} P x$, such that $V\left(\phi\left(t ; 0, x_{0}, u, \sigma\right)\right)$ is strictly decreasing for any fixed nonorigin initial state $x_{0}$.

\section{Lemmas}

Lemma 1: [9] The controllable set of system (1) is the smallest subspace of $\mathbf{R}^{n}$ that is invariant under all $A_{i}$ for $i \in \bar{m}$ and contains all image spaces of $B_{i}$ for $i \in \bar{m}$.

The following two lemmas are adopted from [2], [10].
Lemma 2: A sufficient condition for quadratic stabilizability is that there exist gain matrices $F_{i}, i \in \bar{m}$ such that the matrix pencil

$$
\left\{\sum_{i \in \bar{m}} w_{i}\left(A_{i}+B_{i} F_{i}\right): w_{i} \geq 0, \sum_{i \in \bar{m}} w_{i}=1\right\}
$$

contains a Hurwitz matrix. For the case of $m=2$, the condition is also necessary.

Lemma 3: System (1) is linear feedback stabilizable if there exist gain matrices $F_{i}, i \in \bar{m}$, a natural number $l$, a set of positive real numbers $\left\{h_{1}, \cdots, h_{l}\right\}$, and a set of indices $\left\{j_{1}, \cdots, j_{l}\right\}$, such that matrix

$$
e^{\left(A_{j_{l}}+B_{j_{l}} F_{j_{l}}\right) h_{l}} \cdots e^{\left(A_{j_{1}}+B_{j_{1}} F_{j_{1}}\right) h_{1}}
$$

is Schur.

\section{Main Results}

\section{A. Controllability Does Not Imply Linear Feed- back Stabilizability}

Consider a controllable switched linear system given by

$$
\dot{x}(t)=A_{\sigma} x(t)+B_{\sigma} u(t) .
$$

It is well known that a completely controllable linear timeinvariant system is both linear state feedback stabilizable and quadratically stabilizable. For switched linear systems, the relationship between the controllability and the stabilizability is much more complicated. In the following, we present an example which is completely controllable but not linear state feedback stabilizable. For this, we need a supporting lemma which sets a necessary condition for linear feedback stabilizability.

Lemma 4: Switched linear system (3) is not piecewise linear feedback stabilizable, if for each set of gain matrices $\left\{F_{i}, i \in \bar{m}\right\}$, there exists a one-dimensional subspace $\mathcal{W}$ of $\mathbf{R}^{n}$, such that $\mathcal{W}$ is $\left(A_{i}+B_{i} F_{i}\right)$-invariant and $\left.\left(A_{i}+B_{i} F_{i}\right)\right|_{\mathcal{W}}$ is unstable for all $i \in \bar{m}$.

Proof: As $\mathcal{W}$ is of dimension one, $\left.\left(A_{i}+B_{i} F_{i}\right)\right|_{\mathcal{W}}$ is in fact a scalar matrix with a positive real entry. Since $\mathcal{W}$ is invariant under $\left(A_{i}+B_{i} F_{i}\right)$ for all $i \in \bar{m}$, any state trajectory initialized from $\mathcal{W}$ will remain in the subspace and hence diverge under arbitrary switching signal. This implies that the switched system is not stabilizable by means of gain matrices $F_{i}, i \in \bar{m}$. The assumption of arbitrariness of the gain matrices in the theorem clearly excludes the possibility of the linear state feedback stabilizability of the switched system. $\diamond$

Example 1: For the third-order switched linear control system (3) with

$$
\begin{array}{ll}
A_{1}=\left[\begin{array}{lll}
0 & 0 & 0 \\
1 & 1 & 0 \\
0 & 0 & 1
\end{array}\right] & B_{1}=\left[\begin{array}{l}
1 \\
0 \\
0
\end{array}\right] \\
A_{2}=\left[\begin{array}{lll}
0 & 0 & 0 \\
0 & 1 & 0 \\
1 & 0 & 1
\end{array}\right] & B_{2}=\left[\begin{array}{l}
0 \\
0 \\
0
\end{array}\right]
\end{array}
$$


it can be verified that the system is completely controllable [9].

For any gain matrices $F_{1}$ and $F_{2}$ with

$$
F_{i}=\left[f_{i 1}, f_{i 2}, f_{i 3}\right] \quad i=1,2
$$

it is clear that

$$
A_{1}+B_{1} F_{1}=\left[\begin{array}{ccc}
f_{11} & f_{12} & f_{13} \\
1 & 1 & 0 \\
0 & 0 & 1
\end{array}\right]
$$

and

$$
A_{2}+B_{2} F_{2}=\left[\begin{array}{ccc}
0 & 0 & 0 \\
0 & 1 & 0 \\
1 & 0 & 1
\end{array}\right]
$$

If $f_{12}^{2}+f_{13}^{2} \neq 0$, it can be verified that subspace

$$
\mathcal{W}=\operatorname{span}\left\{\left[\begin{array}{c}
0 \\
-f_{13} \\
f_{12}
\end{array}\right]\right\}
$$

is invariant under $A_{i}+B_{i} F_{i}$ for $i=1,2$. Otherwise, subspace

$$
\mathcal{W}=\operatorname{span}\left\{\left[\begin{array}{l}
0 \\
1 \\
0
\end{array}\right]\right\}
$$

is invariant under $A_{i}+B_{i} F_{i}$ for $i=1,2$. In either case, we have

$$
\left.\left(A_{i}+B_{i} F_{i}\right)\right|_{\mathcal{W}}=1 \quad i=1,2
$$

which are unstable. By Lemma 4, the switched linear control system is not linear feedback stabilizable. $\diamond$

It is interesting to notice that the unstable sub-dynamics (subspace) rely on the (parameters of) gain matrices. In other words, different sequences of gain matrices may correspond to different unstable sub-dynamics. This means that piecewise linear gain matrices are not always able to eliminate unstable common sub-dynamics. A possible way to overcome this is either searching for nonlinear feedback controllers or extending the scheme of piecewise linear feedback stabilization (for example, a subsystem is associated with more than one linear controller). This is an important subject for further investigation.

\section{B. Classification}

As shown in [5], each switched linear control system can be converted into a normal form by means of suitable coordinate change and feedback transformation. Unlike the linear time-invariant case where each controllable system can be converted into the Brunovsky form, the normal forms are not unique for switched systems. As a case study, we consider a controllable third-order switched linear system (3) with two subsystems, i.e., $n=3$ and $m=2$.

For the purpose of stabilizability classification, we only need to consider the case that the system is single-input, that is,

$$
\operatorname{rank} B_{1}+\operatorname{rank} B_{2}=1
$$

Other systems can be converted into single-input by nonregular state feedback as shown in [5]. For a single-input switched system, without loss of generality that $\operatorname{rank} B_{1}=1$ and $B_{2}=0$. Complete controllability implies either of the five cases:

(a) $\operatorname{rank}\left[B_{1}, A_{1} B_{1}, A_{1}^{2} B_{1}\right]=3$;

(b) $\operatorname{rank}\left[B_{1}, A_{1} B_{1}, A_{1}^{2} B_{1}\right]=2$, $\operatorname{rank}\left[B_{1}, A_{1} B_{1}, A_{2} B_{1}\right]=3$

and

(c) $\operatorname{rank}\left[B_{1}, A_{1} B_{1}, A_{1}^{2} B_{1}\right]=\operatorname{rank}\left[B_{1}, A_{1} B_{1}, A_{2} B_{1}\right]=2$, and $\operatorname{rank}\left[B_{1}, A_{1} B_{1}, A_{2} A_{1} B_{1}\right]=3$;

(d) $\operatorname{rank}\left[B_{1}, A_{1} B_{1}\right]=1, \operatorname{rank}\left[B_{1}, A_{2} B_{1}, A_{1} A_{2} B_{1}\right]=3$;

(e) $\operatorname{rank}\left[B_{1}, A_{1} B_{1}\right]=1, \operatorname{rank}\left[B_{1}, A_{2} B_{1}, A_{1} A_{2} B_{1}\right]=2$, and $\operatorname{rank}\left[B_{1}, A_{2} B_{1}, A_{2}^{2} B_{1}\right]=3$.

By means of coordinate change and feedback transformation, the switched system can be converted into the normal form [5] $\Sigma\left(\bar{A}_{i}, \bar{B}_{i}\right)_{\bar{m}}$ with $\bar{B}_{1}=\left[\begin{array}{l}1 \\ 0 \\ 0\end{array}\right]$ and $\bar{B}_{2}=0$. For case (a), pair $\left(A_{1}, B_{1}\right)$ is completely controllable, hence the normal form is

$$
\bar{A}_{1}=\left[\begin{array}{lll}
0 & 0 & 0 \\
1 & 0 & 0 \\
0 & 1 & 0
\end{array}\right] \text { and } \bar{A}_{2}=\left[\begin{array}{ccc}
* & * & * \\
* & * & * \\
* & * & *
\end{array}\right]
$$

where ' $*$ ' denotes the entry which cannot be determined by means of controllability. For case (b), the first two columns of $\bar{A}_{1}$ and the first column of $\bar{A}_{2}$ are fixed, the other column of $\bar{A}_{1}$ is constrained but the other columns of $\bar{A}_{2}$ are totally unspecified. Hence, the matrices are in form

$$
\bar{A}_{1}=\left[\begin{array}{lll}
0 & 0 & 0 \\
1 & 0 & * \\
0 & 0 & *
\end{array}\right] \text { and } \bar{A}_{2}=\left[\begin{array}{ccc}
0 & * & * \\
0 & * & * \\
1 & * & *
\end{array}\right]
$$

Cases (c)-(e) can be discussed in the same way, and the normal forms are

$$
\begin{aligned}
& \bar{A}_{1}=\left[\begin{array}{lll}
0 & 0 & 0 \\
1 & 0 & * \\
0 & 0 & *
\end{array}\right] \text { and } \bar{A}_{2}=\left[\begin{array}{lll}
* & 0 & * \\
* & 0 & * \\
0 & 1 & *
\end{array}\right] \\
& \bar{A}_{1}=\left[\begin{array}{lll}
0 & 0 & 0 \\
0 & 0 & * \\
0 & 1 & *
\end{array}\right] \text { and } \bar{A}_{2}=\left[\begin{array}{lll}
0 & * & * \\
1 & * & * \\
0 & * & *
\end{array}\right] \\
& \bar{A}_{1}=\left[\begin{array}{lll}
0 & 0 & 0 \\
0 & * & * \\
0 & 0 & *
\end{array}\right] \text { and } \bar{A}_{2}=\left[\begin{array}{lll}
0 & 0 & * \\
1 & 0 & * \\
0 & 1 & *
\end{array}\right]
\end{aligned}
$$

respectively.

\section{Quadratic Stabilization}

The following lemma establishes a simple sufficient condition for quadratic feedback stabilizability.

Lemma 5: For a single-input switched system (3), suppose that there exists a sequence of real numbers $w_{i}, i \in \bar{m}$, such that matrix pair $\left(\sum_{i \in \bar{m}} w_{i} A_{i}, B_{1}\right)$ is controllable. Then, the switched system is quadratically stabilizable.

Proof: Without loss of generality, we assume that each $w_{i}$ is nonnegative and $w_{1}>0$ (see Remark 2 below). 
Let $A_{0}=\sum_{i \in \bar{m}} w_{i} A_{i}$ and $B_{0}=w_{1} B_{1}$. As $\left(A_{0}, B_{1}\right)$ is controllable, $\left(A_{0}, B_{0}\right)$ is also controllable. Therefore, we can find a feedback gain matrix $F_{1}$ such that $A_{0}+B_{0} F_{1}$ is Hurwitz. Let $F_{2}=0$. Introducing the piecewise linear state feedback control input

$$
u(t)=F_{\sigma} x(t)
$$

we have that matrix

$$
\sum_{i \in \bar{m}} w_{i}\left(A_{i}+B_{i} F_{i}\right)=A_{0}+B_{0} F_{1}
$$

is Hurwitz. By Lemma 2, the switched system is quadratically stabilizable. $\diamond$

Remark 1: From the proof, the eigenvalues of the average matrix $A_{0}+B_{0} F_{1}$ can be arbitrarily (symmetrically) assigned by appropriately choosing $F_{1}$. On the other hand, the convergence rate of the switched system can arbitrarily approach that of the average system by a suitable switching signal with sufficiently high switching frequency (see, e.g., [10]). As a result, the convergence rate of the switched system can be arbitrarily pre-assigned.

Remark 2: Note that the controllability of pair $\left(\sum_{i \in \bar{m}} w_{i} A_{i}, B_{1}\right)$ for a set $\left\{w_{1}, \cdots, w_{m}\right\}$ implies the controllability of pair $\left(\sum_{i \in \bar{m}} w_{i} A_{i}, B_{1}\right)$ for almost any set $\left\{w_{1}, \cdots, w_{m}\right\}$ in $\mathbf{R}^{m}$. Here "for almost all parameter values" is to be understood as "for all parameter values except for those in some proper algebraic variety in the parameter space". In other words, the set

$$
\left\{\left(w_{1}, \cdots, w_{m}\right):\left(\sum_{i \in \bar{m}} w_{i} A_{i}, B_{1}\right) \text { is not controllable }\right\}
$$

is a variety in $\mathbf{R}^{m}$. This comes from the fact that the controllability is a generic property (see, e.g., [13]). As a result, controllability is preserved in an open and dense set of $\mathbf{R}^{m}$.

It can be verified that, for forms (a), (c), (d) and (e), the condition of Lemma 5 is always satisfied; for forms (b), the condition of Lemma 5 is violated if and only if the normal form is

$$
\bar{A}_{1}=\left[\begin{array}{ccc}
0 & 0 & 0 \\
1 & 0 & 0 \\
0 & 0 & v_{1}
\end{array}\right] \text { and } \bar{A}_{2}=\left[\begin{array}{ccc}
0 & v_{2} & v_{3} \\
0 & v_{4} & 0 \\
1 & -v_{1} & v_{4}
\end{array}\right]
$$

where $v_{1}, \cdots, v_{4}$ are arbitrary real numbers.

For switched system in the form (6), a detailed analysis based on Lemma 2 shows that, the system is quadratically stabilizable if and only if either $v_{1}<0$ or $v_{4}<0$. In other words, the system is not quadratically stabilizable for the case when $v_{1} \geq 0$ and $v_{4} \geq 0$.

Finally, we consider the case that

$$
\operatorname{rank} B_{1}+\operatorname{rank} B_{2} \geq 2 \text {. }
$$

Without loss of generality, we assume that

$$
\operatorname{rank} B_{2} \leq \operatorname{rank} B_{1} \leq 2
$$

Let $b_{1}$ be the first column of $B_{1}$. According to [5], there exist a nonsingular matrix $T$, and feedback gain matrices $F_{1}$ and
$F_{2}$, such that the single-input switched system $\Sigma\left(\bar{A}_{i}, \bar{b}_{i}\right)_{\bar{m}}$ is in the normal form, where

$$
\begin{gathered}
\bar{A}_{i}=T\left(A_{i}+B_{i} F_{i}\right) T^{-1} \quad i=1,2 \\
\bar{b}_{1}=T b_{1}=e_{1} \quad \bar{b}_{2}=0 .
\end{gathered}
$$

Let $\bar{B}_{i}=T B_{i}$ for $i=1,2$. It is clear that the stabilizability of system $\Sigma\left(A_{i}, B_{i}\right)_{\overline{2}}$ is equivalent to the stabilizability of $\Sigma\left(\bar{A}_{i}, \bar{B}_{i}\right)_{2}$. For the latter, it has been proven that the system is quadratically stabilizable except for the case when $\bar{A}_{1}$ and $\bar{A}_{2}$ are in the form (6). As a result, we only need to address this special case.

If rank $B_{1}=2$, then, there is a $\bar{b}_{3} \notin \operatorname{span}\left\{e_{1}\right\}$ such that

$$
\bar{B}_{1}=\left[\bar{b}_{1}, \bar{b}_{3}\right]
$$

It can be verified that, there exist a gain matrix $\bar{F}_{1}$, and nonnegative real numbers $w_{1}$ and $w_{2}$, such that matrix

$$
w_{1}\left(\bar{A}_{1}+\bar{B}_{1} \bar{F}_{1}\right)+w_{2} \bar{A}_{2}
$$

is Hurwitz, which means that system $\Sigma\left(\bar{A}_{i}, \bar{B}_{i}\right)_{\overline{2}}$ is quadratically stabilizable. Similarly, if $\operatorname{rank} B_{1}=\operatorname{rank} B_{2}=1$, it can be verified that, there always exist a gain matrix $\bar{F}_{2}$, and nonnegative real numbers $w_{1}$ and $w_{2}$, such that matrix

$$
w_{1} \bar{A}_{1}+w_{2}\left(\bar{A}_{2}+\bar{B}_{2} \bar{F}_{2}\right)
$$

is Hurwitz, which also means that system $\Sigma\left(\bar{A}_{i}, \bar{B}_{i}\right)_{\overline{2}}$ is quadratically stabilizable.

Summarizing the above analysis, we have the following theorem.

Theorem 1: For a third-order controllable switched linear control system (3) with two subsystems, the system is quadratically stabilizable if and only if either of the following conditions holds:

(i) $\operatorname{rank} B_{1}+\operatorname{rank} B_{2} \geq 2$;

(ii) the system is single-input, and it is not equivalent to normal form (6);

(iii) the system is single-input, and it is equivalent to normal form (6) with either $v_{1}<0$ or $v_{4}<0$.

\section{Non-quadratic Stabilizability}

For the case where the system is not quadratically stabilizable, it may be linear feedback stabilizable as illustrated in the following.

To stabilize systems in the form (6), we seek a linear feedback control input such that the closed-loop system is stabilizable by means of periodic switching signals. By Lemma 3 , it suffices to find a feedback gain vector $f_{1}=$ $\left[f_{11}, f_{12}, f_{13}\right]$, and two positive real numbers $h_{1}$ and $h_{2}$, such that matrix

$$
\exp \left(A_{2} h_{2}\right) \exp \left(\left(A_{1}+b_{1} f_{1}\right) h_{1}\right)
$$

is Schur.

Fix a positive real number $h_{1}$. Let $f_{11}=-2 \rho, f_{12}=$ $-\rho^{2}$, and $f_{13}=\eta$, where $\rho$ and $\eta$ are real numbers to be 
determined. It can be computed that

$$
e^{\left(A_{1}+b_{1} f_{1}\right) h_{1}}=\exp \left(\left[\begin{array}{ccc}
-2 \rho & -\rho^{2} & \eta \\
1 & 0 & 0 \\
0 & 0 & v_{1}
\end{array}\right] h_{1}\right) .
$$

The analytic expression of $\exp \left(A_{2} h_{2}\right)$ can also be computed as

$$
e^{A_{2} h_{2}}=\left[\begin{array}{ccc}
v_{5} & v_{6} & v_{7} \\
0 & e^{v_{4} h_{2}} & 0 \\
v_{8} & v_{9} & v_{10}
\end{array}\right]
$$

where $v_{5}, \cdots, v_{10}$ are analytic functions of $h_{2}$. Note that $v_{1} v_{8}+v_{9}$ is a nonzero function if and only if $v_{2} \neq v_{1} v_{4}$. As the function is analytic, it is nonzero for almost any $h_{2}$ (except for possibly isolated points) when $v_{2} \neq v_{1} v_{4}$.

Suppose that $v_{2} \neq v_{1} v_{4}$. Fix a positive $h_{2}$ such that $v_{1} v_{8}+$ $v_{9} \neq 0$. Let

$$
\eta=\frac{-v_{10}}{v_{1} v_{8}+v_{9}}\left(\rho+v_{1}\right)^{2}
$$

With some manipulation, we can express $\left(A_{2} h_{2}\right) \exp \left(\left(A_{1}+b_{1} f_{1}\right) h_{1}\right)$ in the form

$\Lambda(\rho)=\left[\begin{array}{ccc}q_{1}(\rho) e^{-\rho h_{1}} & q_{2}(\rho) e^{-\rho h_{1}} & \frac{r_{1}+q_{3}(\rho) e^{-\rho h_{1}}}{\left(\rho+v_{1}\right)^{2}} \\ q_{4}(\rho) e^{-\rho h_{1}} & q_{5}(\rho) e^{-\rho h_{1}} & \frac{r_{2}+q_{6}(\rho) e^{-\rho h_{1}}}{\left(\rho+v_{1}\right)^{2}} \\ q_{7}(\rho) e^{-\rho h_{1}} & q_{8}(\rho) e^{-\rho h_{1}} & \frac{q_{9}(\rho) e^{-\rho h_{1}}}{\left(\rho+v_{1}\right)^{2}}\end{array}\right]$

where $r_{1}, r_{2}$ are fixed real numbers and $q_{i}(\cdot), i=1, \cdots, 9$ are polynomials of $\rho$ whose degrees are less than 3 .

It is clear that

$$
\Lambda_{\infty} \stackrel{\text { def }}{=} \lim _{\rho \rightarrow \infty} \Lambda(\rho)=\left[\begin{array}{ccc}
0 & 0 & r_{1} \\
0 & 0 & r_{2} \\
0 & 0 & 0
\end{array}\right]
$$

The spectral radius of this matrix is zero. As a result, for any given positive number $\epsilon<1$, there is a $\rho_{\epsilon}$ such that

$$
\operatorname{sr}(\Lambda(\rho)) \leq \epsilon \quad \forall \rho \geq \rho_{\epsilon},
$$

where sr $A$ denotes the spectral radius of matrix $A$. Pick such a $\rho$, the closed-loop switched system is exponentially stable with the convergence rate not less than $\frac{\ln \epsilon}{h_{1}+h_{2}}$.

Next, we turn to the case that $v_{2}=v_{1} v_{4}$ in form (6). In this case, we can transform system $\sum\left(\bar{A}_{i}, \bar{B}_{i}\right)_{\overline{2}}$ into an equivalent form $\sum\left(\hat{A}_{i}, \bar{B}_{i}\right) \overline{2}$ with

$$
\begin{aligned}
& \hat{A_{1}}=T \bar{A}_{1} T^{-1}+T \bar{B}_{1} f_{1}=\left[\begin{array}{ccc}
0 & 0 & 0 \\
1 & v_{1} & 0 \\
0 & 0 & v_{1}
\end{array}\right] \\
& \hat{A}_{2}=T \bar{A}_{2} T^{-1}=\left[\begin{array}{ccc}
0 & 0 & v_{3} \\
0 & v_{4} & 0 \\
1 & 0 & v_{4}
\end{array}\right]
\end{aligned}
$$

where

$$
T=\left[\begin{array}{ccc}
1 & -v_{1} & 0 \\
0 & 1 & 0 \\
0 & 0 & 1
\end{array}\right] \text { and } f_{1}=\left[\begin{array}{lll}
v_{1} & v_{1}^{2} & 0
\end{array}\right]
$$

For system $\sum\left(\hat{A}_{i}, \bar{B}_{i}\right) \overline{2}$, let

$$
\bar{f}_{1}=\left[-\bar{\rho}_{1}-\bar{\rho}_{2}+v_{1},-\bar{\rho}_{1} \bar{\rho}_{2}, \bar{f}_{13}\right]
$$

where $\bar{\rho}_{1}, \bar{\rho}_{2}$ and $\bar{f}_{13}$ will be determined later. Simple calculation gives

$$
\exp \left(\left(\hat{A}_{1}+\bar{B}_{1} \bar{f}_{1}\right) \bar{h}_{1}\right)=\left[\begin{array}{ccc}
\omega_{1} & \omega_{2} & \omega_{3} \\
\omega_{4} & \omega_{5} & \omega_{6} \\
0 & 0 & 1
\end{array}\right] e^{\bar{h}_{1} v_{1}}
$$

where

$$
\begin{aligned}
\omega_{1} & =\frac{-\bar{\rho}_{2} e^{-\bar{h}_{1} \bar{\rho}_{2}}+e^{-\bar{h}_{1} \bar{\rho}_{1}} \bar{\rho}_{1}}{-\bar{\rho}_{2}+\bar{\rho}_{1}} \\
\omega_{2} & =\frac{\left(e^{-\bar{h}_{1} \bar{\rho}_{1}}-e^{-\bar{h}_{1} \bar{\rho}_{2}}\right) \bar{\rho}_{2} \bar{\rho}_{1}}{-\bar{\rho}_{2}+\bar{\rho}_{1}} \\
\omega_{3} & =-\frac{\bar{f}_{13}\left(e^{-\bar{h}_{1} \bar{\rho}_{1}}-e^{-\bar{h}_{1} \bar{\rho}_{2}}\right)}{-\bar{\rho}_{2}+\bar{\rho}_{1}} \\
\omega_{4} & =-\frac{e^{-\bar{h}_{1} \bar{\rho}_{1}}-e^{-\bar{h}_{1} \bar{\rho}_{2}}}{-\bar{\rho}_{2}+\bar{\rho}_{1}} \\
\omega_{5} & =\frac{-\bar{\rho}_{2} e^{-\bar{h}_{1} \bar{\rho}_{1}}+e^{-\bar{h}_{1} \bar{\rho}_{2}} \bar{\rho}_{1}}{-\bar{\rho}_{2}+\bar{\rho}_{1}} \\
\omega_{6} & =\frac{\bar{f}_{13}\left(-e^{-\bar{h}_{1} \bar{\rho}_{2}} \bar{\rho}_{1}+\bar{\rho}_{2} e^{-\bar{h}_{1} \bar{\rho}_{1}}-\bar{\rho}_{2}+\bar{\rho}_{1}\right)}{\bar{\rho}_{1} \bar{\rho}_{2}\left(-\bar{\rho}_{2}+\bar{\rho}_{1}\right)} .
\end{aligned}
$$

Denote

$$
e^{\hat{A}_{2} \bar{h}_{2}}=\left[\begin{array}{ccc}
\bar{v}_{5} & 0 & \bar{v}_{6} \\
0 & e^{v_{4} \bar{h}_{2}} & 0 \\
\bar{v}_{7} & 0 & \bar{v}_{8}
\end{array}\right] .
$$

Suppose that $v_{3}>0$, then we have $\bar{v}_{8}>e^{v_{4} \bar{h}_{2}}$. Let

$$
\bar{\rho}_{1}=\frac{1}{\bar{h}_{1}} \ln \frac{\bar{v}_{8}-e^{v_{4} \bar{h}_{2}}}{\bar{v}_{8} e^{\bar{v}_{1} \bar{h}_{1}}}
$$

and

$$
\bar{f}_{13}=\frac{1}{\bar{v}_{7}}\left(\bar{v}_{8} e^{v_{1} \bar{h}_{1}}+e^{v_{4} \bar{h}_{2}}\right)
$$

When $\bar{\rho}_{2} \rightarrow \infty$, the spectral radius of matrix

$$
\exp \left(\hat{A}_{2} \bar{h}_{2}\right) \exp \left(\left(\hat{A}_{1}+\bar{B}_{1} \bar{f}_{1}\right) \bar{h}_{1}\right)
$$

approaches zero. As a result, for any given positive number $\epsilon<1$, there is a $\rho_{\epsilon}$ such that for all $\bar{\rho}_{2}>\rho_{\epsilon}$,

$$
\operatorname{sr}\left(\exp \left(\hat{A}_{2} \bar{h}_{2}\right) \exp \left(\left(\hat{A}_{1}+\bar{B}_{1} \bar{f}_{1}\right) \bar{h}_{1}\right)\right) \leq \epsilon .
$$

Pick such a $\bar{\rho}_{2}$, the closed-loop switched system is exponentially stable with the convergence rate not less than $\frac{\ln \epsilon}{\bar{h}_{1}+\bar{h}_{2}}$.

Now, suppose that $v_{3}<-\frac{v_{4}^{2}}{4}$. In this case, let

$$
\bar{f}_{1}=\left[-2 \rho+v_{1},-\rho^{2}, 0\right]
$$

where $\rho$ is a positive real number to be determined. At the same time, let

$$
\bar{h}_{2}=\frac{2}{\sqrt{-4 v_{3}+v_{4}^{2}}}\left(-\arctan \left(\frac{\sqrt{-4 v_{3}+v_{4}^{2}}}{v_{4}}\right) .\right.
$$





Fig. 1. State and input trajectories of system (9)

It can be calculated that matrix $\exp \left(\hat{A}_{2} \bar{h}_{2}\right)$ is of form

$$
\begin{gathered}
{\left[\begin{array}{ccc}
* & 0 & * \\
0 & * & 0 \\
* & 0 & 0
\end{array}\right] \text {. From this, we can establish that }} \\
\lim _{\rho \rightarrow \infty} \exp \left(\hat{A}_{2} \bar{h}_{2}\right) \exp \left(\hat{A}_{1} \bar{h}_{1}\right)=\left[\begin{array}{lll}
0 & 0 & * \\
0 & 0 & 0 \\
0 & 0 & 0
\end{array}\right] .
\end{gathered}
$$

As a result, for sufficiently large $\rho$, the closed-loop system is exponentially stable with any pre-assigned rate of convergence.

When $v_{3}=0$, it can be verified that Lemma 4 applies and hence the system is not linear feedback stabilizable. In the case that $-\frac{v_{4}^{2}}{4} \leq v_{3}<0$, the stabilizability of system (8) is still an open problem.

To summarize, we have the following theorem.

Theorem 2: For a third-order single-input controllable switched linear control system (3) with two subsystems, suppose that its normal form is in the form (6). Then, a sufficient condition for non-quadratic stabilizability is either $v_{2} \neq v_{1} v_{4}$ or $v_{3} \notin\left[-\frac{v_{4}^{2}}{4}, 0\right]$.

\section{E. An Illustrative Example}

Consider controllable single-input normal system given by

$$
\hat{A}_{1}=\left[\begin{array}{lll}
0 & 0 & 0 \\
1 & 1 & 0 \\
0 & 0 & 1
\end{array}\right] \text { and } \hat{A}_{2}=\left[\begin{array}{ccc}
0 & 0 & 2 \\
0 & 1 & 0 \\
1 & 0 & 1
\end{array}\right] \text {. }
$$

This corresponds to the form (8) with $v_{3}>0$.

Let $\bar{h}_{1}=\bar{h}_{2}=1$ and $\bar{\rho}_{2}=500$. From the design procedure described in the last subsection, we have

$$
\bar{f}_{1}=[-498.2269,386.5457,-1078.5935]
$$

It can be verified that matrix $e^{\hat{A}_{1} \bar{h}_{1}} e^{\hat{A}_{2} \bar{h}_{2}}$ has spectral radius 0.2592 and hence is Schur stable.

The above analysis shows that the system is feedback stabilizable. A sample state trajectory and the corresponding input trajectory are shown in Figure 1, where the initial state is $x(0)=[1.4435,-0.3510,0.6232]^{T}$.

\section{Conclusion}

In this work, we examined the underlying relationship between switched controllability and switched stabilizability for switched linear systems. It has been revealed that complete controllability does not imply piecewise linear state feedback stabilizability. As a case study, detailed stability analysis and design have been carried out for third-order switched linear control systems with two subsystems.

\section{References}

[1] R.A. Decarlo, M.S. Branicky, S. Pettersson, and B.Lennartson, "Perspective and results on the stability and stabilizability of hybrid systems," Proceedings of IEEE, vol. 88, no. 7, pp. 1069-1082, 2000.

[2] E. Feron, "Quadratic stabilizability of switched systems via state and output feedback," Technique report, CICS-P-468, MIT, 1996.

[3] B. Hu, X. Xu, P.J. Antsaklis, and A.N. Michel, "Robust stabilizing control laws for a class of second-order switched systems," Sys. Contr. Lett., vol. 38, pp. 197-207, 1999.

[4] Z. Ji, L. Wang, and G. Xie, "New results on the quadratic stabilization of switched linear systems," in: 42nd IEEE CDC, 2003, pp. 16571662.

[5] Z. Sun, "Canonical forms of switched linear control systems," in Proc. Amer. Contr. Conf., Boston, MA, 2004, pp. 5182-5187.

[6] Z. Sun and S.S. Ge, "Dynamic output feedback stabilization of a class of switched linear systems," IEEE Trans. Circ. Syst.-Part I, vol. 50, no. 8, pp. 1111-1115, 2003.

[7] Z. Sun and S.S. Ge, "Analysis and synthesis of switched linear control systems," Automatica, vol. 41, no. 2, pp. 181-195, 2005.

[8] Z. Sun and S.S. Ge, Switched Linear Systems: Control and Design, London: Springer, 2005.

[9] Z. Sun, S.S. Ge, and T.H. Lee, "Reachability and controllability criteria for switched linear systems," Automatica, vol. 38, no. 5, pp. 775-786, 2002.

[10] J. Tokarzewski, "Stability of periodically switched linear systems and the switching frequency," Int. J. Systems Sci., vol. 18, no. 4., pp. 697726, 1987.

[11] M.A. Wicks, P. Peleties, and R.A. DeCarlo, "Construction of piecewise Lyapunov functions for stabilizing switched systems," Proc. 33rd Conf. Decision Control, 1994, pp. 3492-3497.

[12] M.A. Wicks, P. Peleties, and R.A. DeCarlo, "Switched controller synthesis for the quadratic stabilization of a pair of unstable linear systems," European J. Control, vol. 4, no. 2, pp. 140-147, 1998.

[13] W.M. Wonham, Linear Multivariable Control - A Geometric Approach, Berlin: Spinger-Verlag, 1979.

[14] X. Xu and P.J. Antsaklis, "Stabilization of second-order LTI switched systems," Int. J. Control, vol. 73, no. 14, pp. 12611279, 2000. 